\title{
UNA ALTA DIVERSIDAD DE HONGOS MICORRÍZICOS ARBUSCULARES INFLUYE EN LA ABSORCIÓN DE CADMIO Y CRECIMIENTO VEGETATIVO DEL CACAO
}

\author{
Geomar Vallejos-Torres ${ }^{1,2}$, Rubén Ruíz-Valles ${ }^{1}$, César E. Chappa-Santa María ${ }^{2}$, \\ Nery Gaona-Jiménez ${ }^{1}$ y César Marín ${ }^{3}$
}

RESUMEN

\begin{abstract}
El cadmio $(\mathrm{Cd})$ en el suelo es un problema en las plantaciones de cacao en el Perú. El objetivo de este estudio fue evaluar el efecto de la diversidad de hongos formadores de micorrizas arbusculares (HMA) provenientes de diferentes provincias de la Amazonía peruana en la absorción de cadmio y en el crecimiento vegetativo del cacao en condiciones de vivero. Se inocularon semillas germinadas con diferentes especies de HMA, a razón de 1500 esporas, en sustratos constituidos por un volumen de arena media y dos volúmenes de tierra agrícola, con adición de dos niveles de Cd (0 y $\left.5 \mathrm{mg} \cdot \mathrm{kg}^{-1}\right)$. Se evaluaron la altura de planta y área foliar, colonización y micelio de HMA; asimismo, se cuantificó el metal en el suelo y en los tallos de los plantones de cacao. Se empleó un diseño completamente al azar con arreglo bifactorial con ocho tratamientos y ocho réplicas por tratamiento, analizado mediante ANOVA y prueba de medias de Tukey. Los resultados indicaron que las especies de HMA del consorcio de la provincia Mariscal Cáceres, promovieron una mayor altura de planta y área foliar, mientras que las especies del consorcio de Lamas, presentaron mayor reducción de Cd en suelo y tallos. Por lo tanto, la diversidad de especies de HMA asociadas a plantas de cacao podría considerarse una herramienta potencial para inmovilizar el Cd y mejorar el crecimiento en plantas de cacao.
\end{abstract}

Palabras clave adicionales: Biodiversidad, fitorremediación, micorrizas, Theobroma

\begin{abstract}
High genetic diversity in arbuscular mycorrhizal fungi influences cadmium uptake and growth of cocoa plants Soil cadmium (Cd) represents a problem in cocoa farms in Peru. The objective of this study was to evaluate the effect of the diversity of arbuscular mycorrhizal fungi (AMF) from different provinces of the Peruvian Amazon on cadmium uptake and cacao plant growth under nursery conditions. Germinated seeds were inoculated with different AMF species, at a rate of 1,500 spores, in substrates conformed by 1:2 sand and agricultural soil, with two levels of addition of Cd $\left(0\right.$ and $\left.5 \mathrm{mg} \cdot \mathrm{kg}^{-1}\right)$. The height of the plant and leaf area, and colonization and mycelium of AMF were evaluated; likewise, Cd levels were quantified in the soil and the stems of the cocoa seedlings. A completely randomized design with a bifactorial arrangement was used with eight treatments and eight replications per treatment, which were compared by ANOVA and mean separation by Tukey's test. The results indicate that the AMF species from the Mariscal Cáceres province consortium promoted greater plant height and leaf area, while the species from the Lamas consortium presented a greater reduction of cadmium in soil and stems. Therefore, the diversity of AMF species associated with cocoa plants could be considered a potential tool to immobilize Cd and improve growth in cocoa plants.
\end{abstract}

Additional keywords: Biodiversity, mycorrhizae, phytoremediation, Theobroma

\section{INTRODUCCIÓN}

El cacao (Theobroma cacao) es uno de los cultivos de mayor importancia económica en el Perú, siendo la región amazónica de San Martín la que presenta la mayor producción, abarcando

Recibido: Mayo 10, 2021

Aceptado: Octubre 25, 2121

${ }^{1}$ Facultad de Ingeniería ambiental. Universidad Católica Sedes Sapientiae, Lima, Perú. e-mail: gvallejost@gmail.com (autor de correspondencia); ruruva_8757@ hotmail.com; nerygaonajimenez@gmail.com

${ }^{2}$ Facultad de Ciencias Agrarias. Universidad Nacional de San Martín. Morales, Perú. e-mail: cechappa@unsm.edu.pe

${ }^{3}$ Institute of Botany, Czech Academy of Sciences. Pruhonice, Czech Republic y Centro de Invest. e Innov. para el Cambio Climático (CiiCC), Universidad Santo Tomás. Santiago, Chile. e-mail: cesar.marin@postgrado.uach.cl 
alrededor del 37,5 \% en Perú (MINAGRI, 2019). No obstante, existen reportes de la existencia de elevados contenidos de $\mathrm{Cd}$ que podrían causar problemas en el sector agrícola (Florida et al., 2019), cuando se ubica en raíces, hojas, testa y cotiledón (Oliva et al., 2020). Este metal también se ha encontrado en las semillas de cacao (Barraza et al., 2017), particularmente en el clon CCN-51 (Tantalean y Huauya), lo que podría limitar significativamente su comercialización (Aguirre et al., 2021).

El cadmio puede bioacumularse y persistir sin biodegradarse, siendo un precursor de diversos cánceres, estrés oxidativo e inflamación y lesión tisular en humanos (Londoño et al., 2016; Das y Al-Naemi, 2019). Por ende, este elemento constituye una preocupación ambiental importante debido a sus posibles implicaciones para la salud humana (Scaccabarozzi et al., 2020), ya que los productos de cacao de América del Sur en particular, a menudo superan los límites establecidos. Los factores que impulsan la absorción de $\mathrm{Cd}$ no están suficientemente estudiados (Zug et al., 2019) aun cuando existen herramientas biotecnológicas que se podrían aplicar para reducir los contenidos, tal es el caso de la biorremediación con microorganismos (Oliva et al., 2020).

Los géneros fúngicos Aspergillus y Trichoderma muestran eficiencia en la biosorción de Cd (Guerra et al., 2014) al igual que los HMA, que cumplen un rol importante en la bioacumulación y tolerancia de plantas (Yongqiang et al., 2021). Así, estos microorganismos se pueden utilizar para eliminarlo de los suelos contaminados (Han et al., 2021), pues realizan simbiosis con más del $80 \%$ de las plantas vasculares, incluyendo la mayoría de cultivos comerciales (Wipf et al., 2019), reduciendo las concentraciones de metales en los brotes y mejorando la tolerancia en las plantas huésped (Huang et al., 2006). Las estrategias de adsorción e inmovilización de $\mathrm{Cd}$ por hifas de HMA podrían ocurrir tanto en las raíces como en la micorrizósfera, incrementando la resistencia de las plantas a dicho metal (Janeeshma and Puthur, 2020).

Se han realizado pocos estudios sobre el efecto de la diversidad de HMA en la inmovilización de $\mathrm{Cd}$ en cacao peruano. En esta investigación se estudiaron los efectos de la diversidad de HMA nativos extraído de fincas de $T$. cacao de la
Región de San Martín, Perú, incluyendo las especies identificadas genéticamente, Glomus hoi, Acaulospora sp., Glomus sp., Microkamienskia sp., Claroideolomus etunicatum, y Microkamienkia peruviana, sobre la absorción de $\mathrm{Cd}$ y el crecimiento vegetal, con la finalidad de facilitar datos base para la revelación de los mecanismos micorrízicos que mejoran la biorremediación de los cultivares.

\section{MATERIALES Y MÉTODOS}

Área de estudio. El ensayo se llevó a cabo en el vivero de la Universidad Católica Sedes Sapientiae, provincia de Rioja, Región de San Martín, Perú, entre marzo y diciembre de 2020. Geográficamente la provincia se encuentra ubicada entre los paralelos $5^{\circ} 23^{\prime} 30^{\prime \prime}$ y $6^{\circ} 15^{\prime} 00^{\prime \prime}$ de latitud sur y los meridianos $77^{\circ} 05^{\prime} 00^{\prime \prime}$ y $77^{\circ} 45^{\prime} 55^{\prime}$ " longitud oeste, con una temperatura media anual de $22,5^{\circ} \mathrm{C}$ y precipitaciones anuales que oscilan entre los 1000 y $1400 \mathrm{~mm}$.

Preparación de medio de crecimiento y aplicación de dosis de cadmio. El experimento tuvo dos niveles de adición de Cd: 0 y $5 \mathrm{mg} \cdot \mathrm{kg}^{-1}$, en base a cloruro $\left(\mathrm{CdCl}_{2}\right)$ disuelto en agua, el que se mezcló cuidadosamente con el medio de crecimiento, correspondiente a sustrato basado en $1 \mathrm{~L}$ de arena y $2 \mathrm{~L}$ de suelo agrícola, colectado en campo de textura franco arenoso y fertilidad baja. El medio de crecimiento fue pesado en bolsas de polipropileno transparente con dimensiones de 40×30×10 $\mathrm{cm}$ a razón de $3 \mathrm{~kg}$ por bolsa. Posteriormente se adicionó el $\mathrm{Cd}$ y el medio fue secado a pleno sol; se realizaron cuatro removidas cada 15 días, durante dos meses para facilitar la estabilización del contaminante.

Inoculación con hongos micorrízicos arbusculares a semillas germinadas. Las semillas de cacao desinfectadas fueron sembradas en arena esterilizada en autoclave $\left(131^{\circ} \mathrm{C}\right.$ y 0,1 $\mathrm{MPa}$ por 2 horas). Al cabo de 7 días éstas germinaron y mostraron una emergencia uniforme, mayor al $95 \%$ en condiciones controladas. Finalmente, las semillas germinadas se extrajeron y se plantaron en cada macetero, donde se adicionaron 1500 esporas de HMA, para dar origen a la colonización en las raíces de las plantas (Vallejos et al., 2019). Los HMA utilizados en este estudio estuvieron conformados por tres consorcios y un testigo control ( $\sin$ 
micorrizas). El de Rioja (denominación por la procedencia de la provincia en mención) estuvo constituido por las especies Glomus hoi y Acaulospora sp.; el de Lamas por Glomus hoi, Rhizoglomus sp., Diversispora aurantia, y Acaulospora sp.; y el de Mariscal Cáceres por Glomus hoi, Glomus sp., Microkamienskia sp., Claroideolomus etunicatum, y Microkamienkia peruviana.

Parámetros de crecimiento de la planta de cacao, micorrización y determinación de la concentración de cadmio. A los 90 días de iniciado el ensayo se midió la altura de la planta de cacao desde la base del tallo hasta el ápice. La evaluación del área foliar se realizó mediante un muestreo destructivo colocando todas las hojas en un sobre con una superficie oscura, donde fueron fotografiadas (Del Águila et al., 2018).

La tinción de las raíces y de las estructuras formadores de HMA se realizó mediante la técnica propuesta por Phillips y Hayman (1970). Posteriormente, se cuantificó la colonización radical mediante la metodología planteada por Brundrett et al. (1984). Asimismo, se evaluó el micelio extraradical a partir de la colecta de $10 \mathrm{~g}$ de suelo, en tres sectores diferentes de cada macetero.

Para la cuantificación de la longitud de micelio extraradical, las muestras dispuestas en las placas Petri fueron observadas en un microscopio estéreo a 4,5 X y con ayuda de un contómetro se cuantificaron las intersecciones hifa-línea. La cantidad numérica obtenida se trasformó a longitud de micelio por unidad de peso de suelo, utilizando la fórmula propuesta por Newman (1966). Las plantas de cacao fueron cuidadosamente removidas de los maceteros de la cual se recolectaron muestras de suelo de acuerdo a los tratamientos para análisis fisicoquímico y concentración de $\mathrm{Cd}$.

De cada planta se extrajeron tallos para la determinación del cadmio. También se determinó la concentración de Cd en el suelo (EPA 3050B, por flama (U.S. EPA, 1996) y tallos por digestión con $\mathrm{HNO}_{3}$ por espectroscopía de absorción atómica (Jinxiu et al., 2020).

Procesamiento y análisis de datos. Se utilizó un diseño completamente al azar con arreglo factorial de los tratamientos: cuatro consorcios de HMA x 2 niveles de $\mathrm{CdCl}_{2}$. Cada tratamiento tuvo ocho repeticiones, para conformar un ensayo con un total de 64 plantas de cacao.

Para analizar el efecto de los factores y su interacción, los datos fueron sometidos a un análisis de varianza y una prueba de comparación de medias de Tukey con una probabilidad de error del $5 \%$. Todos los análisis fueron realizados empleando el lenguaje de programación $\mathrm{R}$ versión 4,0.2 using R Statistical Software (R Core Team, 2020).

\section{RESULTADOS Y DISCUSIÓN}

Altura y área foliar en plantas micorrizadas de cacao con aplicación de cadmio. El Cuadro 1 muestra que no existió interacción entre los efectos de la inoculación con HMA y adición de $\mathrm{CdCl}_{2}$ al suelo sobre la altura de la planta y el área foliar. Sin embargo, tanto la altura como el área foliar fueron afectados por los HMA y por el cadmio de manera independiente (efectos simples).

Con diferentes niveles de $\mathrm{Cd}$ del suelo con los consorcios micorrízicos, la altura de la planta y el área foliar aumentaron, dentro de determinados límites, en comparación con el tratamiento sin aplicación de HMA (Figura 1). Cuando no se incorporó $\mathrm{Cd}$ en el suelo, la altura de la planta en el tratamiento con HMA procedente de Mariscal Cáceres fue mayor que la de Sin HMA (control) en un 11,54\%; del mismo modo, el área foliar fue mayor en un $21,15 \%$.

Cuadro 1. Valores de $\mathrm{F}$ y probabilidad $(P)$ bidireccionales que examinan los efectos de la inoculación de hongos micorrízicos arbusculares (HMA) e interacción (HMA x Cd) sobre la altura y área foliar de plantas de cacao $\left(\mathrm{cm}^{2}\right)$

\begin{tabular}{|c|c|c|c|c|c|c|}
\hline \multirow{2}{*}{ Variable } & \multicolumn{2}{|c|}{ HMA } & \multicolumn{2}{|c|}{$\mathrm{Cd}$} & \multicolumn{2}{|c|}{ HMA $\times \mathrm{Cd}$} \\
\hline & $\mathrm{F}$ & $P$ & $\mathrm{~F}$ & $P$ & $\mathrm{~F}$ & $P$ \\
\hline Altura de planta & 3,89 & 0,0291 & 50,01 & $\leq 0,0001$ & 0,17 & 0,9165 \\
\hline Área foliar & 10,45 & 0,0005 & 95,47 & $<0,0001$ & 1,49 & 0,2540 \\
\hline
\end{tabular}




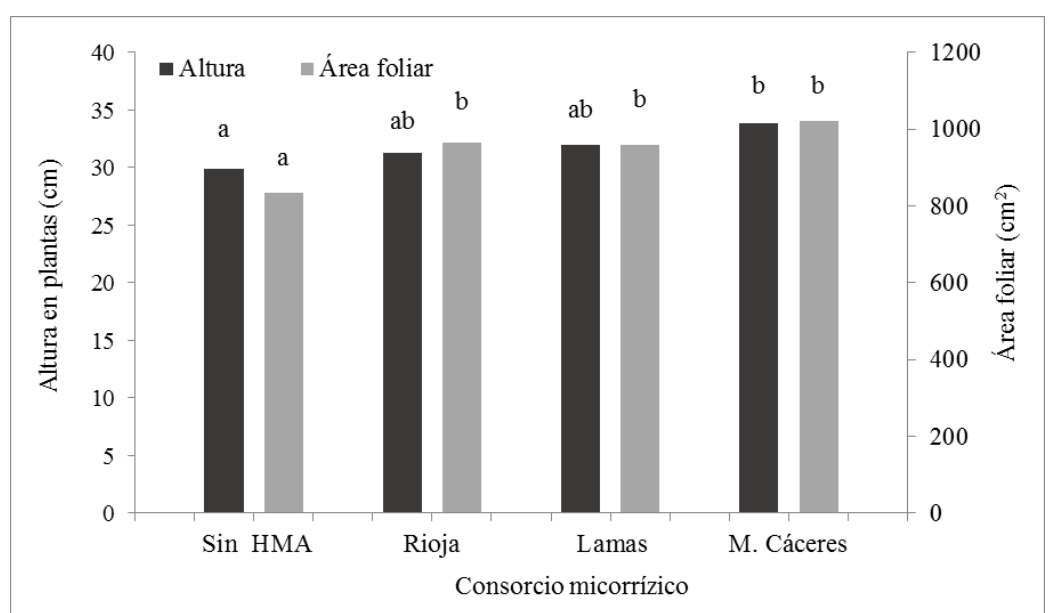

Figura 1. Altura y área foliar de plantas de cacao en función de la inoculación con HMA. Letras sobre las barras indican diferencias significativas según la prueba de Tukey $(P \leq 0,05)$

Cuando el contenido de $\mathrm{Cd}$ en el suelo fue de 5 $\mathrm{mg} \cdot \mathrm{kg}^{-1}$, la altura de la planta en el tratamiento con las especies Glomus hoi, Glomus sp., Microkamienskia sp., Claroideolomus etunicatum, y Microkamienkia peruviana fue mayor que en las plantas sin HMA en un 15,42 \% (Figura 2). Resultados similares lo mostró el área foliar, con un incremento de $20,06 \%$. Por otra parte, como se esperaba, se obtuvo mayor altura y área foliar en aquellas plantas que no recibieron aplicaciones del cloruro de cadmio.

Entre las especies de HMA probadas, las plantas de T. cacao asociadas con Glomus hoi, Glomus sp., Microkamienskia sp., Claroideolomus etunicatum y Microkamienkia peruviana (consorcio Mariscal Cáceres) parecen ser las más tolerantes al Cd. En este estudio se inhibió el crecimiento de plantas de T. cacao, tanto para la altura y como para el área foliar en plantones con suelo contaminado con $\mathrm{CdCl}_{2}$.

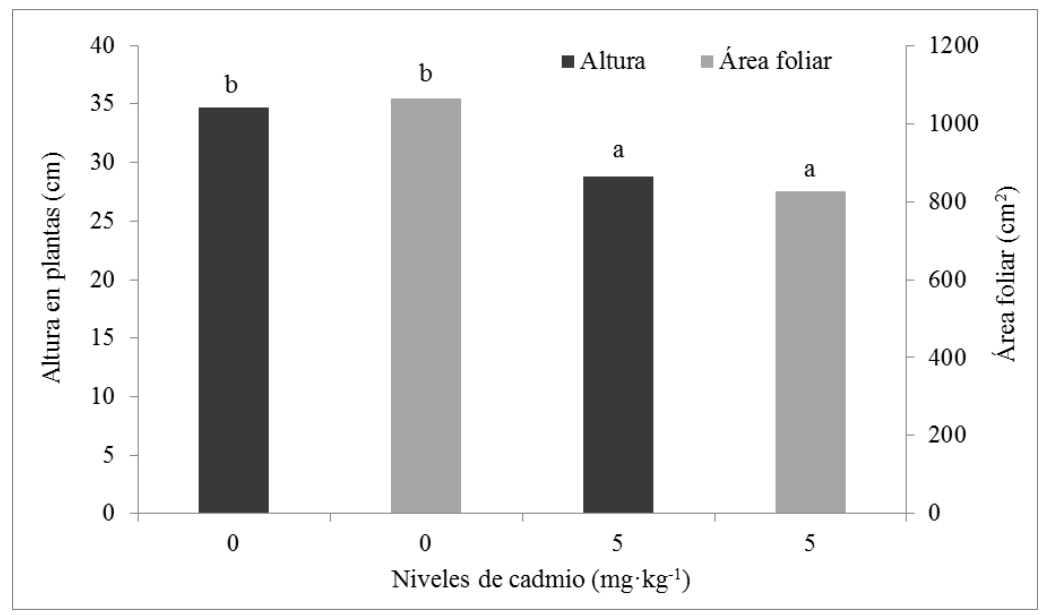

Figura 2. Altura y área foliar de plantas de cacao en función del nivel de cadmio. Letras sobre las barras indican diferencias significativas según la prueba de Tukey $(P \leq 0,05)$

Ambas variables disminuyeron con el aumento del contenido del metal en el suelo. Sin embargo, en aquellos que no tuvieron aplicación de cadmio el crecimiento vegetal aumentó en comparación con el tratamiento con $5 \mathrm{mg} \cdot \mathrm{kg}^{-1}$, lo que indicó que dicho elemento provocó primero el bloqueo del crecimiento y del desarrollo de las raíces de las plantas de T. cacao. Algunos estudios han demostrado que los HMA pueden disminuir la concentración de $\mathrm{Cd}$ en las plantas (Redon et al., 2008; Sun et al., 2018; Fengge et al., 2019) al aumentar su biomasa. Como se presenta en este estudio, el crecimiento de las plantas de T. cacao se redujo significativamente al aumentar la 
concentración de $\mathrm{Cd}$, lo que concuerda con lo indicado por Hassan et al. (2013).

Los HMA tienen en general un efecto positivo sobre el crecimiento vegetal bajo estrés por metales pesados, lo cual fue corroborado por Garg y Kaur (2013) y Aguilera et al. (2017).

Colonización $(\%)$ y micelio en plantas de cacao micorrizadas con aplicación de cadmio. El Cuadro 2 muestra diferencias significativas para las variables de colonización micorrízica y micelio extraradical en plantas de cacao.

$\mathrm{Al}$ no incorporar $\mathrm{Cd}$ en el suelo, la colonización micorrízica de los consorcios de Rioja y Lamas alcanzaron el 89,33\% y 84,09\%, respectivamente (Figura 3). En este mismo tratamiento control, la longitud de micelio de los consorcios de Mariscal Cáceres y Lamas fue de $9,21 \mathrm{~cm}$ y $8,23 \mathrm{~cm}$, respectivamente (Figura 4).

Cuadro 2. Valores de $\mathrm{F}$ y probabilidad $(P)$ bidireccionales que examinan los efectos de la inoculación de hongos micorrízicos arbusculares (HMA) e interacción (HMA x Cd) sobre la colonización micorrízica $(\%)$ y micelio extraradical $(\mathrm{cm})$

\begin{tabular}{|c|c|c|c|c|c|c|}
\hline \multirow{2}{*}{ Variable } & \multicolumn{2}{|c|}{ HMA } & \multicolumn{2}{|c|}{$\mathrm{Cd}$} & \multicolumn{2}{|c|}{ HMA x Cd } \\
\hline & $\mathrm{F}$ & $P$ & $\mathrm{~F}$ & $P$ & $\mathrm{~F}$ & $P$ \\
\hline Colonización micorrízica & 114,55 & $<0,0001$ & 2,56 & 0,1290 & 1,52 & 0,2477 \\
\hline Longitud del micelio & 73,47 & $<0,0001$ & 2,7 & 0,9590 & 5,62 & 0,0080 \\
\hline
\end{tabular}

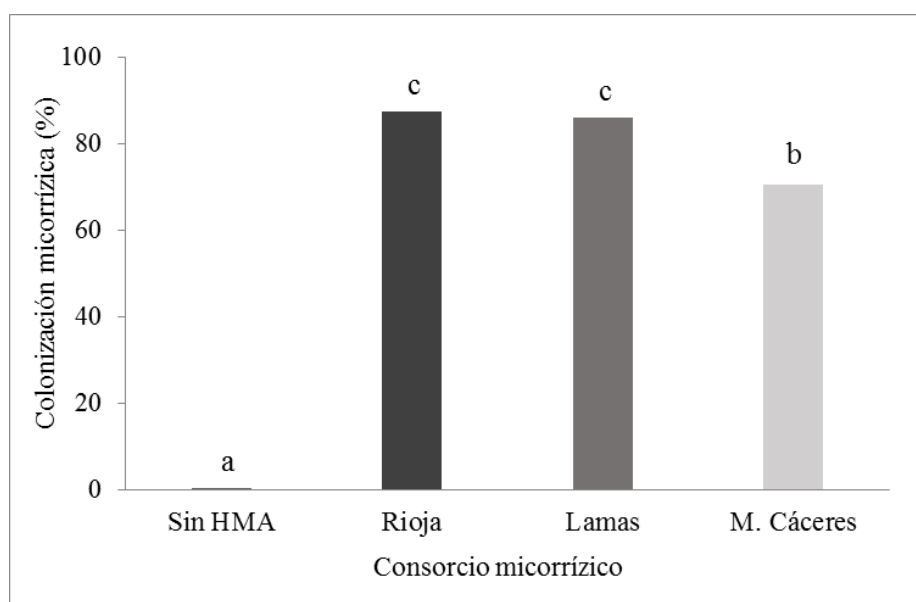

Figura 3. Colonización de plantas de cacao en función de la inoculación de HMA. Letras sobre las barras indican diferencias significativas según la prueba de Tukey $(P \leq 0,05)$

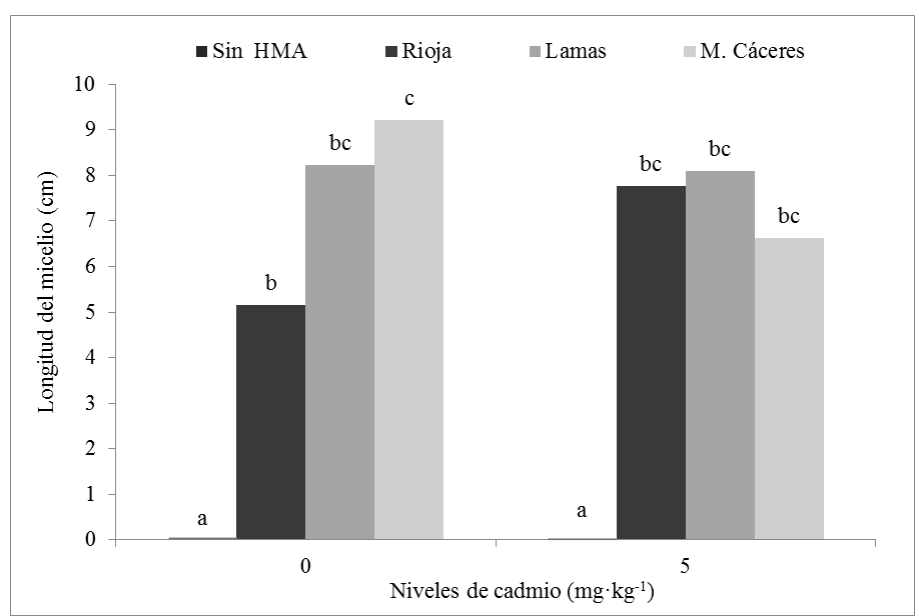

Figura 4. Longitud del micelio extraradical en plantas de cacao en función de los consorcios micorrízicos y niveles de cadmio. Letras sobre las barras indican diferencias significativas según la prueba de Tukey $(P \leq 0,05)$ 
En este estudio la inhibición de la colonización de raíces por HMA fue observada después de la aplicación del cadmio, siendo en primer orden el consorcio Rioja seguido de Lamas y Mariscal Cáceres, similar a los resultados obtenidos por Miransari (2017). Tanto la colonización como la longitud del micelio coinciden con los estudios realizados por Pawlowska y Charvat (2004) y Janoušková y Pavlíková (2010).

La adición de $\mathrm{Cd}$ afectó diferencialmente la colonización y la longitud de micelio de acuerdo a la diversidad de especies entre los consorcios. Este resultado corrobora los de Blaudez et al. (2000) quienes señalan que los metales pesados inhibieron el crecimiento de los hongos ectomicorrízicos.

Contenido de cadmio en suelo y tallos de plantas micorrizadas de cacao. El Cuadro 3 muestra la significancia del tratamiento con $\mathrm{Cd}$ sobre su contenido en el tallo de la planta, así como el efecto del metal y los HMA sobre el contenido en el suelo, sin efecto de interacciones. En la Figura 5 se observa que con $5 \mathrm{mg} \cdot \mathrm{kg}^{-1} \mathrm{de} \mathrm{Cd}$ el contenido de este elemento mostró diferencias entre los HAM, donde los consorcios Lamas y Mariscal Cáceres exhibieron una reducción del metal en suelo de 8,66 y $6,30 \%$, respectivamente (Figura 5). En contraste, cuando se aplicó el elemento, el contenido en el suelo y tallos de las plantas se incrementó (Figura 6).

Cuadro 3. Valores de $\mathrm{F}$ y probabilidad $(P)$ bidireccionales que examinan los efectos de la inoculación de hongos micorrízicos arbusculares (HMA), Cd en el suelo $(\mathrm{Cd})$ e interacción (HMA x Cd) sobre el contenido de cadmio en el suelo y en los tallos

\begin{tabular}{ccccccccc}
\hline \multirow{2}{*}{ Variable } & \multicolumn{2}{c}{ HMA } & & \multicolumn{2}{c}{ Cd } & & \multicolumn{2}{c}{ HMA x Cd } \\
\cline { 2 - 3 } \cline { 8 - 9 } \cline { 8 - 9 } & F & $P$ & & F & $P$ & & F & $P$ \\
\hline Contenido de Cd en suelo & 4,43 & 0,0190 & & 972,29 & $<0,0001$ & & 1,39 & 0,2829 \\
Contenido de Cd en tallo & 1,03 & 0,4056 & & 1247,03 & $<0,0001$ & & 2,44 & 0,1023 \\
\hline
\end{tabular}

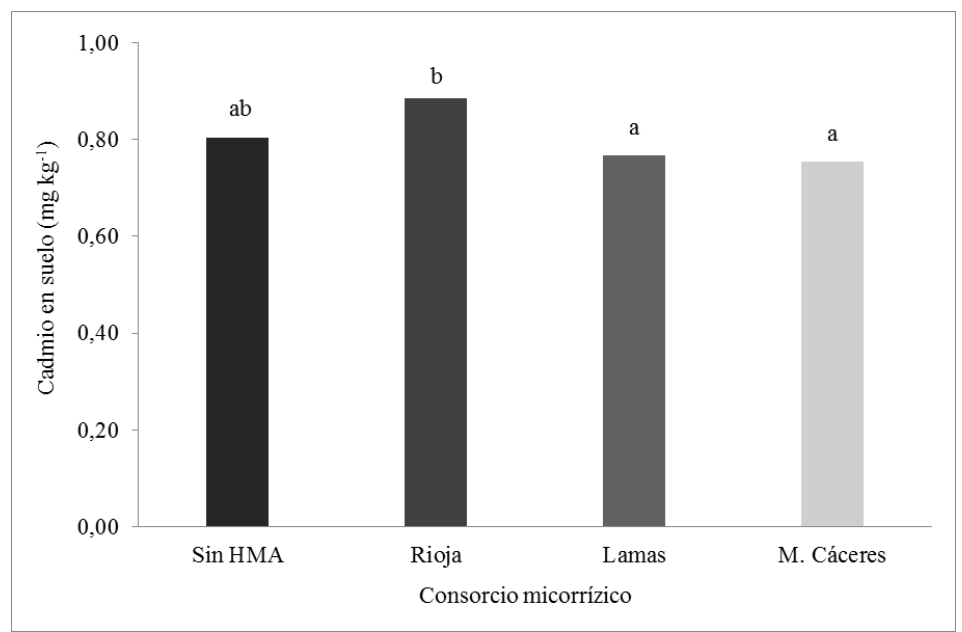

Figura 5. Cadmio en suelos con plantas de cacao en función de la inoculación con HMA. Letras sobre las barras indican diferencias significativas según la prueba de Tukey $(P \leq 0,05)$

Los contenidos de Cd en tallos de plantas de cacao micorrizadas disminuyeron, lo que podría atribuirse a mecanismos fisiológicos de los HMA al aumentar la tolerancia de las plantas huésped a la toxicidad de metales pesados (Wang et al., 2013; Li et al., 2016). Se pudo observar que a mayor diversidad de especies de HMA también existió una mayor reducción de $\mathrm{Cd}$. Las asociaciones de micorrizas pueden contribuir a reducir la transferencia de metales pesados a las plantas actuando como una barrera de exclusión (Cabral et al., 2015).

Las posibles razones de la capacidad de los consorcios micorrízicos de Lamas y Mariscal Cáceres para absorber $\mathrm{Cd}$ podría deberse al mayor contenido de especies y esporulación extrarradical 
del micelio y también al alto intercambio de solutos en la interfase hongo - célula raíz (arbúsculos) bajo concentraciones elevadas de metales (Pawlowska y Charvat, 2004).

La influencia de los HMA en la absorción de metales de las plantas varía según las concentraciones de éstos (Zhang et al., 2015). Se pudo comprobar que los hongos micorrízicos redujeron la acumulación de $\mathrm{Cd}$ en los tallos de plantas de cacao, presumiblemente mediante el aumento de la retención del metal dentro de las raíces (Wang et al., 2020).

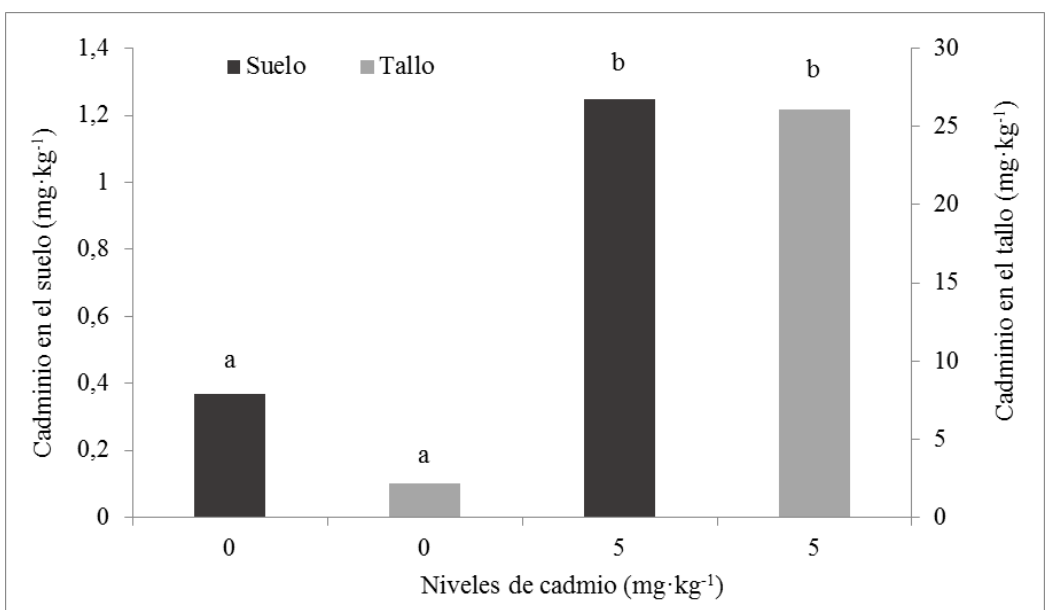

Figura 6. Nivel de cadmio en el suelo y tallos de plantas de cacao en función de la aplicación del metal pesado. Letras sobre las barras indican diferencias significativas según la prueba de Tukey $(P \leq 0,05)$

\section{CONCLUSIONES}

Las micorrizas procedentes de la provincia de Mariscal Cáceres conformadas por las especies Glomus hoi, Glomus sp., Microkamienskia sp., Claroideolomus etunicatum y Microkamienkia peruviana, mostraron los mayores incrementos en altura y área foliar en las plantas de cacao y a su vez presentaron las mayores reducciones de $\mathrm{Cd}$, tanto en el suelo como en los tallos.

El consorcio procedente de la provincia de Lamas conformado por las especies Glomus hoi, Rhizoglomus sp., Diversispora aurantia, Acaulospora sp. y el de Rioja conformado por Glomus hoi y Acaulospora sp., mostraron los mayores incrementos en colonización y longitud de micelio extraradical. La diversidad de especies de hongos micorrízicos arbusculares asociados a plantas podría convertirse un potencial para inmovilizar el $\mathrm{Cd}$ y mejorar el crecimiento morfológico en plantas de cacao.

\section{AGRADECIMIENTO}

La Universidad Católica Sedes Sapientiae reconoce el apoyo financiero del proyecto
Concytec-Banco Mundial "Mejoramiento y Ampliación de los Servicios del Sistema Nacional de Ciencia, Tecnología e Innovación Tecnológica" 8682-PE, a través de su unidad ejecutora Fondecyt, en el marco de la actividades del proyecto "Biorremediación con micorrizas arbusculares nativas en el control de cadmio de clones de Theobroma cacao como estrategia sostenible para la seguridad alimentaria ecológica en la Amazonía peruana" con Contrato 105-2018FONDECYT-BM-IADT-AV.

\section{LITERATURA CITADA}

1. Aguilera P., C. Marín, F. Oehl, R. Godoy, F. Borie y P.E. Cornejo. 2017. Selection of aluminum tolerant cereal genotypes strongly influences the arbuscular mycorrhizal fungal communities in an acidic Andosol. Agriculture, Ecosystems and Environment 246: 86-93.

2. Aguirre-Forero, S.E., N.V. PiranequeGambasica y J.R. Vásquez-Polo. 2021. Contenido de metales pesados en suelos y tejidos de cacao en el departamento del Magdalena, Colombia: énfasis en cadmio. Entramado 16(2): 298-310. 
3. Barraza F., E. Schreck, E. Schreck, T. Lévêque, G. Uzu, F. López, J. Ruales, J. Prunier, A. Marquet y L. Maurice. 2017. Cadmium bioaccumulation and gastric bioaccessibility in cacao: a field study in areas impacted by oil activities in Ecuador, Environ. Pollut. 229: 950-963.

4. Blaudez D., C. Jacob, K. Turnau, J.V. Colpaert, U. Ahonen-Jonnarth, R. Finlay et al. 2000. Differential responses of ectomycorrhizal fungi to heavy metals in vitro. Mycol Res 104: 1366-1371.

5. Brundrett, M.C., Y. Piche y R.L. Peterson. 1984. A new method for observing the morphology of vesicular-arbuscular mycorrhizae. Can. J. Bot. 62: 2128-2134.

6. Cabral L., C.R.F. Sousa, A.J. Giachini y J.O. Siqueira. 2015. Arbuscular mycorrhizal fungi in phytoremediation of contaminated areas by trace elements: mechanisms and major benefits of their applications. Word J. Microbiol. Biotechnol. 31(11): 1655-1664.

7. Das, S.C. y H.A. AL-Naemi. 2019. Toxicidad por cadmio: estrés oxidativo, inflamación y lesiones tisulares. Enfermedades Profesionales y Medicina Ambiental 7(4): 144-163.

8. Del Águila, K.M., G. Vallejos-Torres, L.A. Arévalo y A.G. Becerra. 2018. Inoculación de consorcios micorrícicos arbusculares en Coffea arabica, variedad Caturra en la región San Martín, Información Tecnológica 29(1): 137146.

9. Fengge, Z., L. Moha, L. Yang, Ch. Yeye y X. Yan. 2019. Effects of arbuscular mycorrhizal fungi, biochar and cadmium on the yield and element uptake of Medicago sativa. Science of the Total Environment. 655: 1150-1158.

10.Florida, N., J. Paucar, S.S. Jacobo y F. Escobar-Mamani. 2019. Efecto de compost y NPK sobre los niveles de microorganismos y cadmio en suelo y almendra de cacao. Journal of High Andean Research 21(04): 264273.

11.Garg, N. y H. Kaur. 2013. Impact of cadmiumzinc interactions on metal uptake, translocation and yield in pigeonpea genotypes colonized by arbuscular mycorrhizal fungi. Journal of Plant Nutrition. 36(1): 67-90.
12.Guerra-Sierra, B.E., A. Sandoval-Meza, L.S. Manrique-González y S.P. Barrera-Rangel. 2014. Ensayos preliminares in vitro de biosorción de cadmio por cepas fúngicas nativas de suelos contaminados. Innovaciencia Facultad Cienc. Exactas Fis. Naturales 2(1): 53-58.

13.Han, Y., O.K. Zveushe, F. Dong, Q. Ling, Y. Chen, S. Sajid et al. 2021. Unraveling the effects of arbuscular mycorrhizal fungi on cadmium uptake and detoxification mechanisms in perennial ryegrass (Lolium perenne). Sci. Total Environ. 798: 149222.

14.Hassan, S.E., M. Hijri y M. St-Arnaud. 2013. Effect of arbuscular mycorrhizal fungi on trace metal uptake by sunflower plants grown on cadmium contaminated soil. N. Biotechnol. 30: 780-787

15.Huang, H, S. Zhang, C. Bao-Dong, W. Naiying, S. Xiao-Quan y P. Christy. 2006. Uptake of atrazine and cadmium from soil by maize (Zea mays L.) in association with the arbuscular mycorrhizal fungus Glomus tunicatum. J. Agr. Food Chem. 54: 93779382.

16.Janeeshma, E. and J.T. Puthur. 2020. Direct y indirect influence of arbuscular mycorrhizae on enhancing metal tolerance of plants. Arch Microbiol. 202(1): 1-16.

17.Janoušková, M. y D. Pavlíková. 2010. Cadmium immobilization in the rhizosphere of arbuscular mycorrhizal plants by the fungal extraradical mycelium. Plant and Soil 332(1): 511-520.

18.Jinxiu, Z., S. Lin, Y. Kai, L. Mingrui, H. Yongmei, Z. Yanqun, Z. Fangdong y L. Tao. 2020. An arbuscular mycorrhizal fungus increased the macroaggregate proportion and reduced cadmium leaching from polluted soil, International Journal of Phytoremediation 22: 1-9.

19.Li, H., N., L.J. Luo. H.M. Zhang, Y.W. Zhao, Q.Y. Li, M.H. Cai, M.H. Wong and C.H. Mo. 2016. Do arbuscular mycorrhizal fungi affect cadmium uptake kinetics, ¿subcellular distribution and chemical forms in rice?. Sci Total Environ. 571: 1183-90

20.Londoño-Franco, L.F, P.T. Londoño-Muñoz y 
F.G. Muñoz-García. 2016. Los riesgos de los metales pesados en la salud humana y animal. Biotecnología en el Sector Agropecuario y Agroindustrial 14(2): 145-153.

21.MINAGRI (Ministerio de Agricultura y Riego). 2019. Plan Nacional de Cultivos. Campaña Agrícola 2019-2020 Reporte de precio y mercado de cacao, Lima, Perú. https://n9.cl/c2wa (consulta de octubre 5, 2021).

22.Miransari, M. 2017. Arbuscular mycorrhizal fungi and heavy metal tolerance in plants. In: Wu QS. (eds.). Arbuscular Mycorrhizas and Stress Tolerance of Plants. Springer, Singapore. 147-161.

23.Newman, E.I. 1966. A Method of estimating the total length of root in a sample. Journal of Applied Ecology 3(1): 139-145.

24. Oliva M., K. Rubio, M. Epquin y S. Marlo Gand Leiva. 2020. Cadmium uptake in native cacao trees in agricultural lands of Bagua, Peru. Agronomy 10(10): 1551.

25.Pawlowska, T.E. y I. Charvat. 2004. Heavymetal stress and development patterns of arbuscular mycorrhizal fungi. Appl. Environ. Microbiol. 70(11): 6643-6649.

26.Phillips, J.M. y D.S. Hayman. 1970. Improved procedures for clearing roots and staining parasitic and vesicular-arbuscular mycorrhizal fungi for rapid assessment of infection, Transactions of the British Mycological Society 55(1): 158-161.

27.Redon, P.O., T. Beguiristain y C. Leyval. 2008. Influence of Glomus intraradices on $\mathrm{Cd}$ partitioning in a pot experiment with Medicago truncatula in four contaminated soils. Soil Biol. Biochem. 40: 2710-2712.

28. $\mathrm{R}$ Core Team. 2020. R: A language and environment for statistical computing. $\mathrm{R}$ Foundation for Statistical Computing, Vienna, Austria. https://www.R-project.org/ (consulta de septiembre 30, 2021).

29. Scaccabarozzi, D., L. Castillo, A. Aromatisi, L. Milne, A. Búllon y M. Muñoz-Rojas. 2020. Soil, site, and management factors affecting cadmium concentrations in cacao-growing soils. Agronomy 10(6): 806.
30.Sun, H., Y. Xie, Y. Zheng, Y. Lin y F. Yang. 2018. La mejora por hongos micorrízicos arbusculares de la capacidad de remediación del $\mathrm{Cd}$ y los factores relacionados con la calidad de la bioenergía de cinco cultivares de pasto varilla en suelos contaminados con $\mathrm{Cd}$. PeerJ, 6, e4425.

31.Tantalean, E. y M. A. Huauya. 2017. Distribución del contenido de cadmio en los diferentes órganos del cacao CCN-51 en suelo aluvial y residual en las localidades de Jacintillo y Ramal de Aspuzana. Revista de Investigación de Agroproducción Sustentable 1(2): 69-78.

32.U.S. EPA. 1996. Method 3050B: Acid Digestion of Sediments, Sludges, and Soils, Revision 2. Washington, DC. https://n9.cl/76t47 (consulta de octubre 5, 2021).

33. Vallejos-Torres, G., T. Sánchez, M.A. García, M. Trigoso y L.A. Arévalo. 2019. Efecto de hongos formadores de micorrizas arbusculares en clones de café (Coffea arabica) variedad Caturra. Acta Agronómica 68(4): 278-284.

34.Wang, F., S. Zhang, P. Cheng, S. Zhang y Y. Sun. 2020. Effects of soil amendments on heavy metal immobilization and accumulation by maize grown in a multiple-metalcontaminated soil and their potential for safe crop production. Toxics 8(4): 102 (6 p.).

35.Wang, F.Y., Z.Y. Shi, X.F. Xu, X.G. Wang y Y.J. Li. 2013. Contribution of AM inoculation and cattle manure to lead and cadmium phytoremediation by tobacco plants. Environ Sci. Process Impacts 15(4): 794-801.

36.Wipf, D., F. Krajinski, D. van Tuinen, G. Recorbet y P.E. Courty. 2019. Trading on the arbuscular mycorrhiza market: from arbuscules to common mycorrhizal Networks. New Phytologist 223(3): 1127-1142.

37. Yongqiang, Y., W. Li, J. Chang, W. Gen, M. Fang, W. Yujiao y Y. Dongguang. 2021. Effects of arbuscular mycorrhizal fungi on the growth and toxic element uptake of Phragmites australis (Cav.) Trin. ex Steud under zinc/cadmium stress. Ecotoxicology and Environmental Safety 213: 112023.

38.Zhang, X., B. Chen y R. Ohtomo. 2015. Mycorrhizal effects on growth, $\mathrm{P}$ uptake and 
Cd tolerance of the host plant vary among different AM fungal species. Soil Science and Plant Nutrition 61(2): 359-368.

39.Zug, K.L., H.A. Huamaní, F. Meyberg, F.
Meyberg y J.S. Cierjacks. 2019. Cadmium accumulation in peruvian cacao (Theobroma cacao L.) and opportunities for mitigation. Water Air Soil Pollution 230(3): 72-80. 